P0046

\title{
COLOUR PREFERENCE IS DEPENDENT ON COLOUR TEMPERATURE, CHROMA AND ILLUMINANCE LEVELS - EXPERIMENTS AND ANALYSIS
}

\author{
Quang Vinh Trinh et al.
}

DOI 10.25039/x46.2019.PO046

from

CIE x046:2019

Proceedings

of the

29th CIE SESSION

Washington D.C., USA, June 14 - 22, 2019

(DOI 10.25039/x46.2019)

The paper has been presented at the 29th CIE Session, Washington D.C., USA, June 14-22, 2019. It has not been peer-reviewed by CIE.

(C) CIE 2019

All rights reserved. Unless otherwise specified, no part of this publication may be reproduced or utilized in any form or by any means, electronic or mechanical, including photocopying and microfilm, without permission in writing from CIE Central Bureau at the address below. Any mention of organizations or products does not imply endorsement by the CIE.

This paper is made available open access for individual use. However, in all other cases all rights are reserved unless explicit permission is sought from and given by the CIE.

CIE Central Bureau

Babenbergerstrasse 9

A-1010 Vienna

Austria

Tel.: +43 17143187

e-mail: ciecb@cie.co.at

www.cie.co.at 


\title{
COLOUR PREFERENCE IS DEPENDENT ON COLOUR TEMPERATURE, CHROMA AND ILLUMINANCE LEVELS - EXPERIMENTS AND ANALYSIS
}

\author{
Vinh, Q.T. ${ }^{1}$, Bodrogi, P. ${ }^{1}$, Khanh, T.Q. ${ }^{1}$ \\ ${ }^{1}$ Technical University of Darmstadt, Laboratory of Lighting Technology, Darmstadt, GERMANY \\ vinh@lichttechnik.tu-darmstadt.de
}

DOI 10.25039/x46.2019.PO046

\begin{abstract}
Colour fidelity (CF) was studied by comparing between test and reference light sources (LSs) based on colour differences. Colour saturation (CS) was discussed with Colour Gamut or Chroma Difference. Colour preference $(C P)$ played the middle role of these colour metrics regarding asymmetric combinations of $C F$ and $C S$. Some studies in this approach were carried out from Flattery Index (Judd, 1967) to CQS Qp (Ohno et at., 2010). However, all of them was not operated well in full considerations of correlated colour temperatures $(C C T)$ and illuminance levels $\left(E_{\mathrm{v}}\right)$. Therefore, in this work the visual experiments are applied and analysed for describing $C P$ under different $C C T$ and $E_{\mathrm{v}}$. New multi - dimension - correlations are discussed operatively in order to establish the new direction of an available $C P$. As a result, the progress from the colour quality $(C Q)$ into lighting quality $(L Q)$ is conducted as the essential demand of modern lighting systems.
\end{abstract}

Keywords: Colour Preference, Colour Quality, Light Quality

\section{Motivation}

Natural light sources, what are familiar with human life, cannot serve themselves in the condition of night and dark environments. Incandescent lamps of Thomas Elva Edison helped to open the night life, but the spectral distribution cannot satisfy the human demand of the beauty of lighted objects. The improvement of fluorescent lamps mainly served to improve the energy efficient, although colour metrics including correlated colour temperature and colour rendering index $(C R I)$ have been also accounted in some extents. Nowadays, the modern solid state lighting system with semiconductor coloured LEDs, phosphor converted LEDs and hybrid multi-channel LED-luminaires can fulfil nearly all diversified aspects of static and dynamic human demands on both colour quality and light quality. Therefore, the scientific requirements of having appropriate colour metrics and lighting metrics are issued naturally. Then, the traditional colour metrics, what based on only unique $C F$, must be modernized appropriately and the frequent discussion of $C S$ should be more deeply operated. The visual experiments and corresponding computations should be implemented as parallel so that possible issues can be processed for more available and applicable solutions of the metrics. In this work, the visual experiments under specific illuminances, CCTs and chroma levels and the mathematical formulations will be described for moving gradually from the colour quality into the light quality for relevant evaluations of modern lighting systems.

\section{The description of visual experiment procedures, computations and optimizations}

In the framework of the experiments, the young students of the Technical University Darmstadt experienced under the lighting conditions of three CCTs (warm white $3000 \mathrm{~K}$, neutral white $4100 \mathrm{~K}$ and daylight white $5600 \mathrm{~K}$ ) of a hybrid multi-channel LED-luminaire that can be programmed for its absolute spectrum with different CCTs, illuminances and chroma enhancement levels. The test colour samples (TCSs) in the experiments are described in Figure 1. Herewith, both standard Macbeth Colour Checker and familiar coloured objects (such as pullover, paintings and others) are applied together under the observation of test persons. The unique question is, how high the preference level of the test person is, based on observing the coloured objects under the lighting conditions of different correlated colour temperatures, chroma enhancement and illuminance levels. The answer of test persons is expressed in the 
semantics scale that was designed like the description in Figure 2. This scale was gained from the study of Brückner in [2].
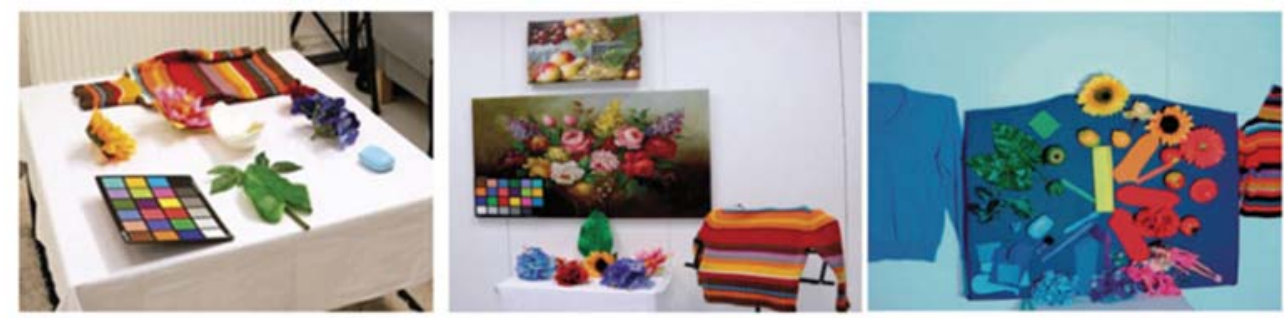

Figure 1 - Colour samples in visual tests under different CCTs, chroma and Illuminances

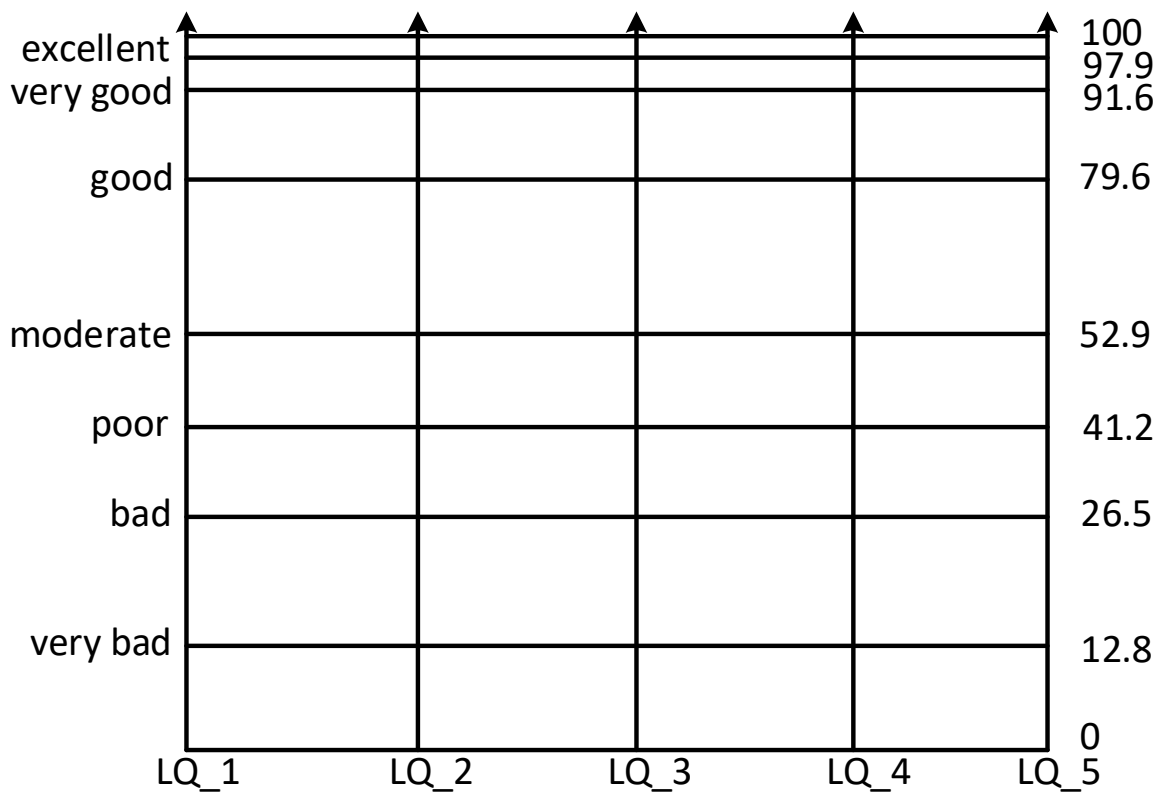

Figure 2 - The semantics scale for the test persons in experiments ( $L Q$ is a light source)

The procedure with the interactive actions between the visual experiments, synthesises and optimizations are described in details in Figure 3. Herewith, it can be recognized that the full power hybrid LED-luminaire with the programmable changes of its spectra, illuminances and colour properties is applied, the test colour samples are available and determined previously and the test persons are selected appropriately so that the second databank of visual evaluations can be achieved.

As well, the complex computations based on the scientific achievements of CIE TC-01-09 with its calculations of the reference light sources and the available scale for IES TM-30-15, the colour space CIE CAM-UCS-02, the concept (Colour Fidelity $R_{\mathrm{f}}$ ) of IES TM-30-15 and the referent results of the visual experiments studied in the literatures [3], [4], [5] and [6] as the start points of the implement help to have the first databank from mathematical computations.

The first databank and the second databank are applied together into the optimization procedures in order to achieve parameters of the mathematic system of the computing equations so that the difference between visual evaluation ratings and computed values is minimized. If the result is not good enough, the quality of the first and second databank should be checked and improved appropriately. When the examination achieves good results, the visual verification with new experiments should be implemented in order to verify for the achieved parameters. When the verification can prove that the parameters are already available, the procedure can be stopped. Otherwise, the procedure must be repeated from the beginning to the end so that the most available results can be achieved. 


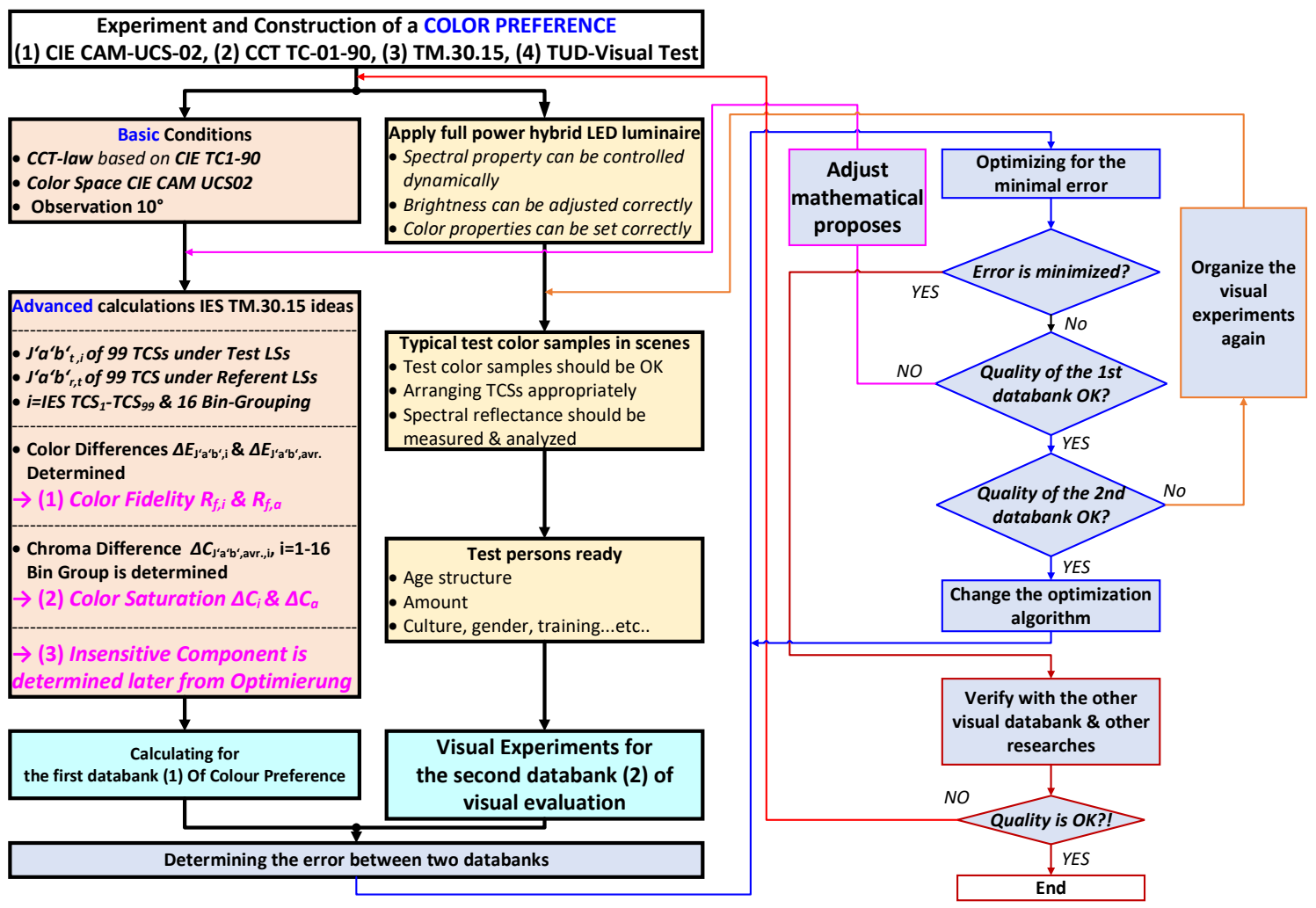

Figure 3 - The procedure for constructing the databanks of visual experiments and computations and optimizing $\left(\Delta C_{\mathrm{a}}\right.$ and $R_{\mathrm{f}, \mathrm{a}}$ are the average values of $\Delta C_{\mathrm{i}}$ and $\left.R_{\mathrm{f}, \mathrm{i}}\right)$

\section{Colour preference under different colour temperatures, chroma and illuminance levels}

The most available results achieved from the procedure described in Section 2 above are used for establishing the three dimensional correlation of the visual evaluation with illuminance and chroma enhancement levels under three lighting conditions of warm white $(W W)$, neutral white $(N W)$ and cold white $(C W)$ like the description in the figures 4,5 and 6 . The coefficients of the determinations denoted $R^{2}$ (" $R$ squared") are highly $0,86,0,95$ and 0,90 corresponding with the cases of $W W, N W$ and $C W$, respectively.

Based on the achievements described in these figures, it can be recognized that although the absolute levels of the computed models are different, but their mathematical formats are similar. These computations can be listed as follows,

$$
\begin{aligned}
& \text { Visual Evaluation }=a \cdot \ln (\text { Illuminance }+b)+c \cdot T C D_{\mathrm{a}}{ }^{2}+d \cdot T C D_{\mathrm{a}}+e \\
& \Delta \boldsymbol{a}_{\text {relative,avr, }, \mathrm{i}}^{\prime}=\frac{a_{\mathrm{t}, \mathrm{avr}, \mathrm{i}}^{\prime} a_{\mathrm{r}, \mathrm{avr}, \mathrm{i}}^{\prime}}{\sqrt{a_{\mathrm{r}, \mathrm{avr}, \mathrm{i}}^{\prime}+b_{\mathrm{r}, \text { avr }, \mathrm{i}}^{\prime}}} \\
& \Delta \boldsymbol{b}_{\text {relative,avr,i }}^{\prime}=\frac{b_{\mathrm{t}, \mathrm{avr}, \mathrm{i}}^{\prime}-\boldsymbol{b}_{\mathrm{r}, \mathrm{avr}, \mathrm{i}}^{\prime}}{\sqrt{\boldsymbol{a}_{r, \text { avr }, i}^{\prime}+b_{r, \text { avr }, i}^{\prime}}} \\
& \Delta C_{\mathrm{i}}=\Delta C_{\mathrm{a}^{\prime} \mathbf{b}^{\prime}, \mathrm{i}, \text { relative }}=\Delta \boldsymbol{a}_{\text {relative,avr, } \mathrm{i}}^{\prime} \cdot \cos \left(\gamma_{\mathrm{BIN}, \mathrm{i}}\right)+\Delta \boldsymbol{b}_{\text {relativ,avr,i }}^{\prime} \cdot \sin \left(\gamma_{\mathrm{BIN}, \mathrm{i}}\right) \\
& \text { Total Chroma Difference }=T C D_{\mathrm{a}}=\sum_{i=1}^{16} \Delta C_{\mathrm{a}^{\prime} \mathrm{b}^{\prime}, \mathrm{i}, \text {,elative }}
\end{aligned}
$$

where $\boldsymbol{a}_{\mathrm{t}, \mathbf{a v r}, \mathbf{i}}^{\prime}, \boldsymbol{b}_{\mathrm{t}, \mathbf{a v r}, \mathbf{i},}^{\prime} \boldsymbol{a}_{\mathbf{r}, \mathbf{a v r}, \mathbf{i},}^{\prime} \boldsymbol{a}_{\mathbf{r}, \text { avr }, \mathbf{i}}^{\prime}$ are the average values of the parameters $\left(a_{\mathrm{t}}^{\prime}, b_{\mathrm{t}}^{\prime}\right.$ calculated on Color Space CIE CAM-UCS-02) and ( $a_{r}^{\prime}, b_{\text {r }}^{\prime}$ calculated on Color Space CIE CAM-UCS-02) of the test colour samples under test and reference light sources, respectively. They are classified into 16 IES TM-30-15 groups ( $\mathrm{i}=1-16)$ with the angle $\gamma_{B I N, i}$ that is determined on the following binning principle, 


$$
\vartheta=\operatorname{rem}\left(\left(\operatorname{atan2}\left(b_{\mathrm{r}}^{\prime}, a_{\mathrm{r}}^{\prime}\right)\right), 2 \cdot \pi\right) \text { if } \vartheta<0 \text { then } \vartheta=2 \cdot \pi+\vartheta
$$

$(6$

$$
N r_{\text {BIN }}=f l o o r\left(\left(\frac{\vartheta}{\pi}\right) \cdot 16\right)+1
$$

$a_{\mathrm{j}}^{\prime}, b_{\mathrm{j}}^{\prime}, J_{\mathrm{j}}^{\prime}$ classified into group $i\left(N r_{\cdot \mathrm{BIN}}=i=1 \rightarrow 16\right)$

$$
\text { Angle } \gamma_{\mathrm{BIN}, \mathrm{i}}=-\frac{\pi}{16}+\frac{\pi}{8} \cdot N r_{\cdot \mathrm{BIN}}(i=1 \rightarrow 16)
$$

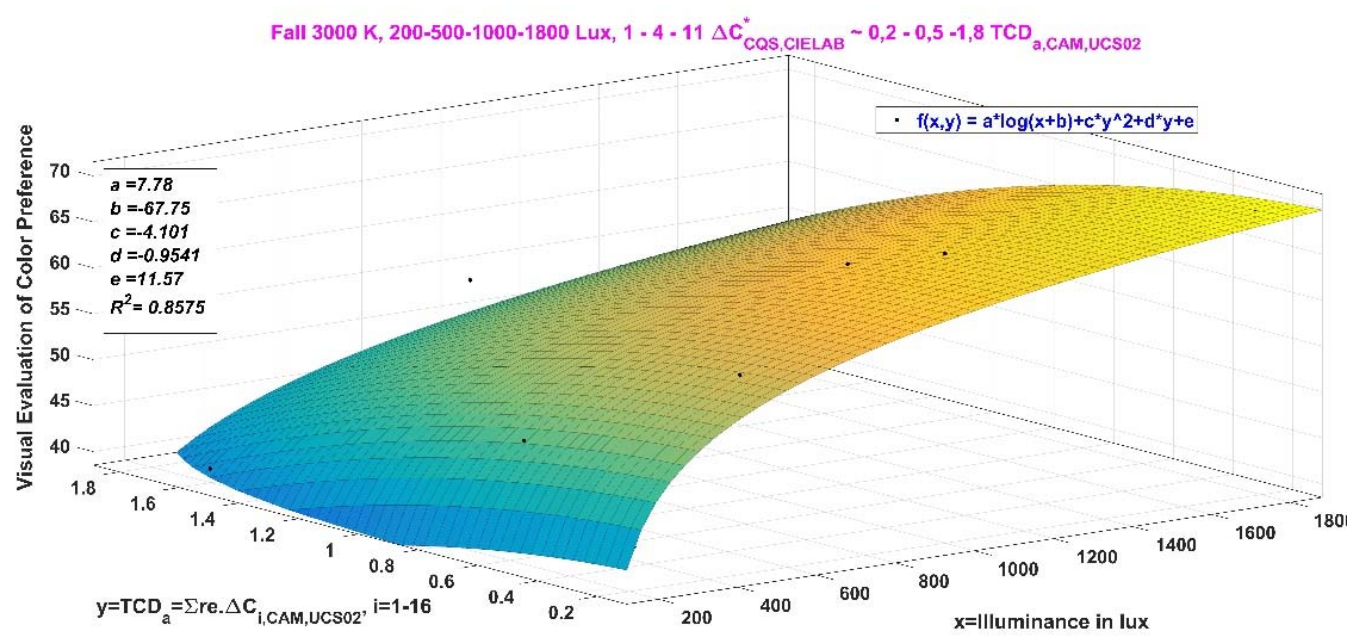

Figure 4 - Three-dimensional correlation of illuminance, chroma enhancement and visual evaluation in the case of the warm white hybrid LED-luminaire $(3000 \mathrm{~K})$

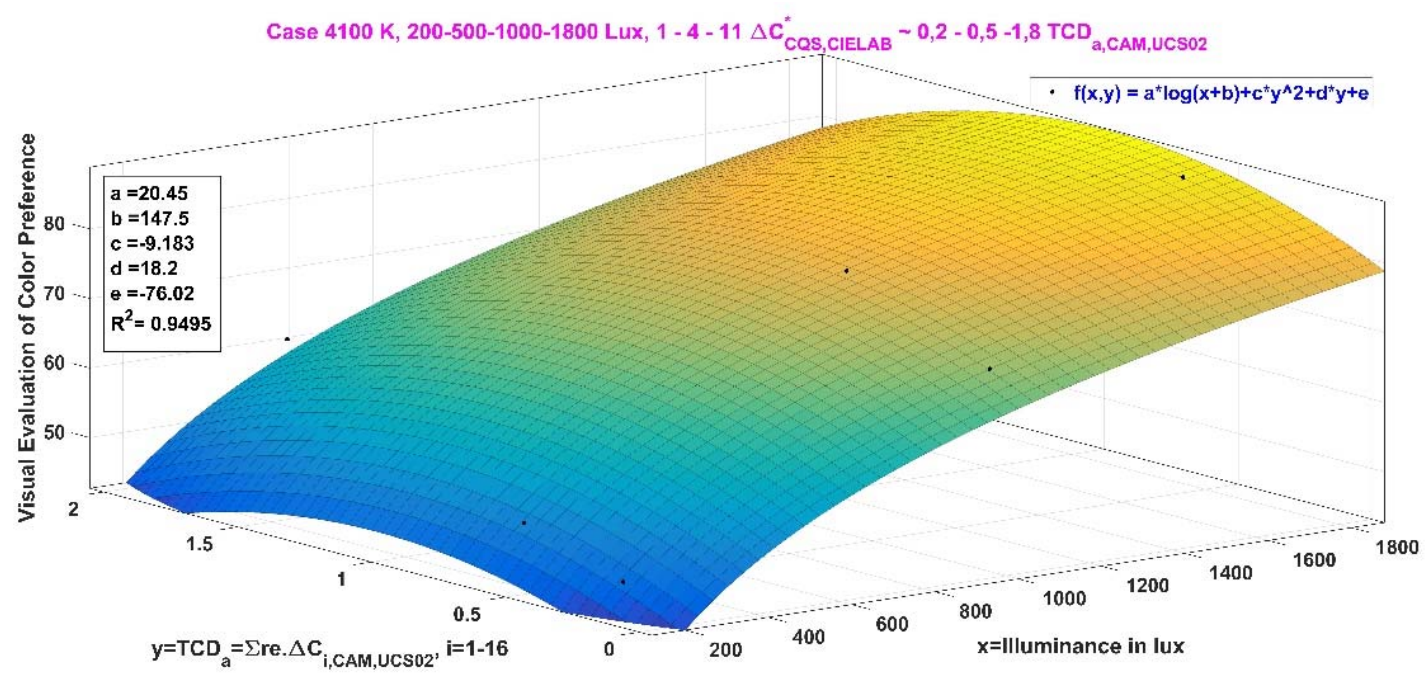

Figure 5 - Three-dimensional correlation of illuminance, chroma enhancement and visual evaluation the case of the neutral white hybrid LED-luminaire ( $4100 \mathrm{~K})$ 


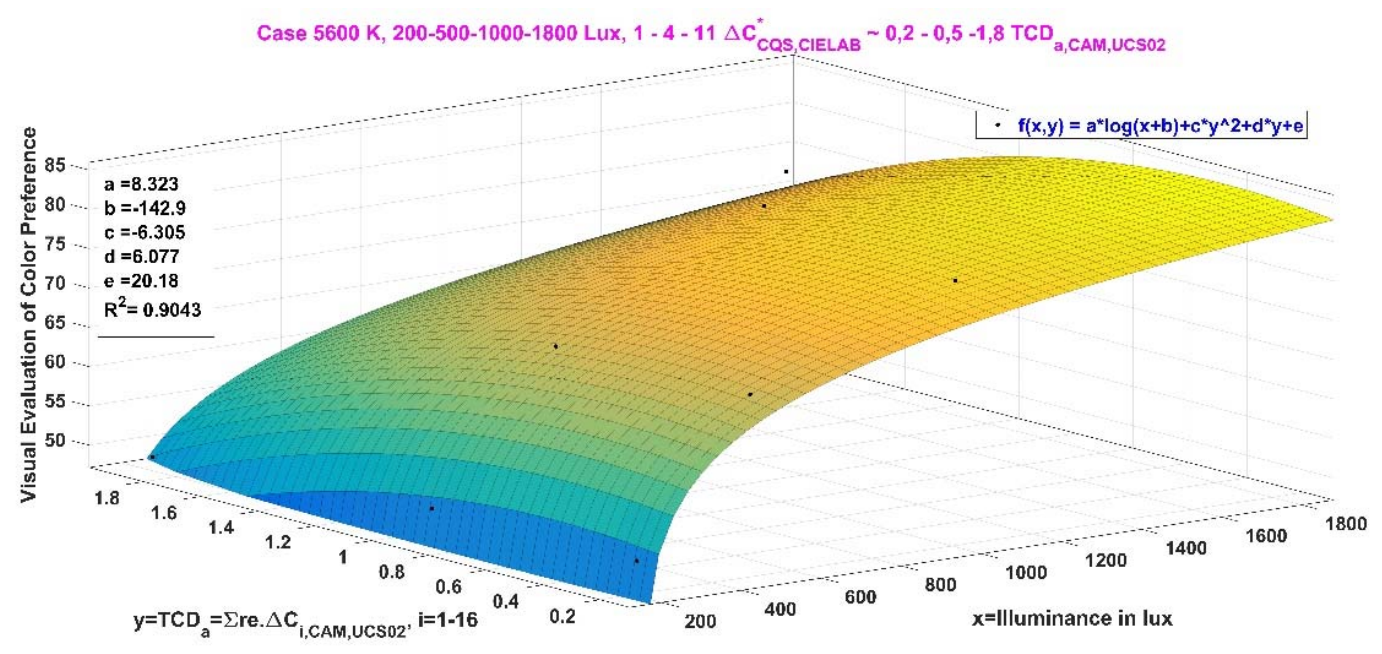

Figure 6 - Three-dimensional correlation of illuminance, chroma enhancement and visual evaluation the case of the cold white hybrid LED-luminaire (5600 K)

The results in the figures 4,5 and 6 prove clearly that the visual evaluation of colour preference is not only dependent on colour fidelity classically and chroma enhancement sophisticatedly, but also on the correlated colour temperatures and illuminance levels. Mathematically, the colour fidelity and chroma enhancement are two aspects of the same issue (one is for the colour difference $\left(\Delta E=\sqrt{\left(a_{\mathrm{t}}^{\prime}-a_{\mathrm{r}}^{\prime}\right)^{2}+\left(b_{\mathrm{t}}^{\prime}-b_{\mathrm{r}}\right)^{2}+\left(J^{\prime}{ }_{\mathrm{t}}-J^{\prime}{ }_{\mathrm{r}}\right)^{2}}\right.$ typically in Colour space CIE CAMUCS02) and the other for the chroma difference $\left(\Delta C=\sqrt{\left(a_{\mathrm{t}}^{\prime}+b_{\mathrm{t}}^{\prime}\right)^{2}}-\sqrt{\left(a_{\mathrm{r}}^{\prime}+b_{\mathrm{r}}^{\prime}\right)^{2}}\right.$ typically in Colour Space CIE CAM-UCS02)) between test and referent sources. Between them, there is a mathematical relationship. In addition, the chroma enhancement value is even limited by the allowance of colour discrimination. If the chroma enhancement is too high, the colour contrast and colour identify of coloured objects under lighting systems are reduced.

Whereas, correlated colour temperature and illuminance are other issues and quite independent on the classical parameters. As well, its preference level is also dependent on the factors of culture, geography, education, career and other human aspects.

\section{Conclusion}

The results of this work clearly identified that the visual evaluation of the human perception is not only dependent on the colour fidelity, but also on chroma enhancement, correlated colour temperature and illuminance. As well, the total chroma difference $\left(T C D_{\mathrm{a}}\right)$ in the mentioned computation can play a good role for describing the chroma enhancement, mathematically.

Based on the results achieved herewith, the evaluation of light sources following only test colour samples and consequently colour quality is out of date and has to be renovated into the new format of light quality, where the illuminance, correlated colour temperature, colour difference, chroma difference and even hue difference should support each other for a complete perception of the quality of light sources and lighting quality which are more available, practical and effective in the practical applications of human-oriented lighting systems.

\section{References}

[1]. JUDD, D. B. 1967. A flattery index for artificial illuminants., Illum. Eng. 62. 593 - 598.

[2]. BRÜCKNER, S. 2014. Farbdifferenz - Skalierung zur Farbqualitätsbeurteilung von HalbleiterLichtquellen, Dissertation, TU Darmstadt. 
[3]. Khanh, T. Q.; Bodrogl, P.; Guo, X.; VINH, Q. T.; FISHER, S. 2017. Color preference, naturalness, vividness and color quality metrics, Part 5: A colour preference experiments at $2000 \mathrm{Ix}$ in a real room, Lighting Research and Technology, DOI: 10.1177/1477153517737133

[4]. Khanh, T. Q.; Bodrogl, P.; VINH, Q. T.; Guo, X.; ANH, T. T. 2017. Color preference, naturalness, vividness and color quality metrics, Part 4: Experiments with still life arrangement at different correlated color temperatures, Lighting Research and Technology, DOI:10.1177/1477153517700 705

[5]. Khanh, T. Q.; BodrogI, P.; VInH, Q. T.; StojanoviC, D. 2016. Color preference, naturalness, vividness and color quality metrics, Part 2: Experiments in a viewing booth and analysis of the combined dataset, Lighting Research and Technology, DOI:10.1177/1477153516643570

[6]. Khanh, T. Q.; BodrogI, P.; VINH, Q. T.; StojanoviC, D. 2016. Color preference, naturalness, vividness and color quality metrics, Part 1: Experiments in a room, Lighting Research and Technology, DOI:10.1177/1477153516643359

[7]. DAVIS, W.; OHNO, Y. 2010. Color quality scale, Optical Engineering, 49: 033602. 